\title{
10 Frugivores, Seeds and Genes: Analysing the Key Elements of Seed Shadows
}

\author{
P. JORDANO
}

\section{Introduction}

A seed shadow is the spatial distribution of all seeds dispersed from an individual plant (Janzen, 1970; Nathan and Muller-Landau, 2000; Schupp et al., 2002). The sum of seed shadows across all individuals in a population plus those seeds dispersed from other populations makes up seed rain. For vertebrate-dispersed species, seed shadows are the primary outcome of plant-frugivore interactions, yet we know very little about how different frugivore species contribute to them. For instance, a few frugivore species might contribute disproportionately to long-distance dispersal (LDD) events, while another subset of dispersers might contribute to the local, short-distance dispersal (see Dennis and Westcott, Chapter 9, this volume). In addition, seed delivery to specific microhabitats might be provided by only a few species out of the whole frugivore assemblage (Reid, 1989; Wenny and Levey, 1998; Jordano and Schupp, 2000; Jordano et al., 2007).

For years, studies of seed dispersal have been severely limited in their potential to characterize seed shadows and to assess the contribution different species of frugivore make to their extent and reach in distance and space (i.e. the dispersal kernels; see Portnoy and Willson, 1993; Clark et al., 1999; Bullock and Clarke, 2000; Nathan, Chapter 11, this volume). The main difficulty has been to succeed in assigning a tree source for a dispersed seed and then link that information with both the species of frugivore that delivered the seed and the subsequent establishment success for the seedling. These are the three basic pieces of information that we need to account for seed shadows: source of seeds, dispersal vector, and establishment outcome.

Variations in seed shadow pattern and extent between individual plants in a population can be large, and the summed seed shadows can generate very complex patterns of seed rain. Marked peaks and troughs in 2-D seed 
density patterns (i.e. the two-dimensional spatial distribution of seeds on the ground), including extensive areas with no seed rain at all, are all possible, resulting in an enormous range of recruitment patterns (see Kwit et al., Chapter 19, this volume). It is the interaction between frugivore foraging and the structure of complex landscapes that generates these peaks and troughs in the seed shadows, marking 'hot-spots' and empty spots of seed rain and, consequently, potential plant recruitment (see Carlo et al., Chapter 16 , this volume). Our present understanding of these complex dynamics is extremely poor, despite unprecedented advances in recent years, especially when we refer to the influences on genetic structure or the relevance of LDD events (see Hardesty, Chapter 12, this volume). The seed shadow is the immediate outcome of interactions with frugivores and represents the first stage of recruitment; it is therefore of paramount importance to understand whether this 'signal' of the interaction with frugivores lasts to the final stage of adult recruitment.

Recent advances in field techniques (e.g. use of radioactive markers, radio-tracking; Primack and Levy, 1988; see Dennis and Westcott, Chapter 9, this volume), molecular genetics tools (e.g. hypervariable simplesequence DNA repeats - SSRs or microsatellites) (Godoy and Jordano, 2001; see Hardesty, Chapter 12, this volume), and GIS-based techniques (Loiselle and Blake, 1993) allow a thorough analysis of seed shadows (Nathan and Muller-Landau, 2000). Thus, several recent studies have marked an extraordinary advance in our ability to overcome long-standing methodological limitations in seed dispersal research (Nathan 2006). These advances fall into two distinct approaches to analysing seed shadows: (i) a 'source tree' perspective and (ii) a 'target or seed delivery site' perspective. Future advances in the field will most probably stem from the combination of the two approaches, as they are complementary rather than exclusive.

The deconstruction of a seed shadow involves two steps:

1. working from the source tree and proceeding away from it by determining dispersal distances and inferring which frugivore species contribute the dispersal events (the 'seed shadow' analysis in its strict sense)

2. working from the microhabitat patches where seeds arrive and inferring which frugivore species contribute the seed rain from where.

Focal observations at fruiting trees have been routine in seed dispersal studies since the late 1970s (Howe and Kerckhove, 1980; Herrera and Jordano, 1981; Snow and Snow, 1988). The simple approach of watching a tree and attempting to infer where seeds go on the basis of the animals removing them implicitly emphasized a 'from the source tree' perspective and led to the tracking of the movement of seeds away from maternal trees. Therefore, tracking methods, either direct or indirect, have been widely used to assess seed shadows and understand the processes generating them: direct mapping of frugivore locations (Julliot, 1997; Whitney et al., 1998; Holbrook et al., 2002; Mack and Druliner, 2003); direct observations of frugivore foraging and seed delivery (Reid, 1989; 
Gómez, 2003; see Stevenson, Chapter 15, this volume); combining frugivore tracking with gut retention times (Fleming, 1988; Murray, 1988; Sun et al., 1997; Westcott and Graham, 2000; Westcott et al., 2005); and using inert markers to track seed movement (Mack, 1995; Vander Wall, 2000; Levey et al., 2005), among others.

On the other hand, a number of studies have used the 'target or seed delivery site' perspective by emphasizing seed rain analysis (Izhaki et al., 1991; Houle, 1992; Nakashizuka et al., 1995; Kollmann and Goetze, 1997; Clark et al., 1999; Alcántara et al., 2000; Harms et al., 2000; Jordano and Schupp, 2000; Rey and Alcántara, 2000; Muller-Landau et al., 2002; Clark et al., 2004; McEuen and Curran, 2004). These studies grew from the analysis of seed banks, but extended this work to encompass the arrival of seeds (Schupp et al., 1989). Work with seed-trap designs has significantly increased our understanding of seed rain patterns and of the initial template from which regeneration processes and recruitment unfold. Additionally, the specific design of the seed-trap monitoring might infl uence the robustness and precision of seed shadow and dispersal kernel estimation (Kollmann and Goetze, 1997; Skarpaas et al., 2005). However, inferences about the causal processes for the observed seed rain patterns remain elusive unless a direct connection between process and pattern can be constructed by combining direct watches at the fruiting trees and seedtrap monitoring (Jordano and Schupp, 2000).

Both approaches to deconstruct seed shadows are methodologically limited by their ability to determine the source tree for dispersed seeds, especially for medium- and long-distance dispersal events and when these involve seed delivery from other populations. Frugivore tracking methods invariably fail to characterize the frequency and extent of extreme LDD events; and even for shorter dispersal, the precise location of the animals when delivering seeds cannot be established (Westcott et al., 2005). Interpretation of seed-trap methods can be limited when the number of potential source trees is large or when seed shadow overlap is extensive (Godoy and Jordano, 2001); additional limitations relate to the specific spacing patterns of the seed traps relative to source trees and the spatial design (Jones et al., 2005; Skarpaas et al., 2005).

In this chapter, I analyse the main components of seed shadows and discuss how hypervariable molecular markers can be used to assess them and the advantages and disadvantages involved in doing so. I present a brief discussion of seed shadow analysis, some illustrative data with Prunus mahaleb, and an analysis of perspectives to link frugivore foraging and seed shadow patterns. Despite enormous recent advances in our understanding of seed shadows and dispersal, many exciting aspects remain underinvestigated. Some of these include the lasting consequences of frugivore foraging on genetic structure in the plant populations, the frequency and extent of LDD events, and the survival prospects of propagules from LDD events. 


\section{Analysing seed shadows}

Fruiting trees are key elements in plant-frugivore interactions because they are the source of dispersed seeds, but they also shape the foraging movements of frugivores, are sinks for dispersed seeds and hot-spots for pathogens and post-dispersal seed predators (Schupp et al., 2002; Clark et al., 2004; see Carlo et al., Chapter 16, this volume; see Gallery et al., Chapter 22, this volume). Their role as seed sources has traditionally been assessed from a tree's perspective; attempting to track the direction and distance the seeds are dispersed. When we consider the opposite view, from the target patch receiving dispersed seeds, different frugivores contribute in different ways to the seed rain arriving at given patches, in terms of dispersal distance, proportion of long-distance dispersal events, and diversity of contributing mother trees (Westcott et al., 2005, see Dennis and Westcott, Chapter 9, this volume; see Nathan, Chapter 11, this volume). This may generate extensive variation in the makeup of the seed shadows, both in terms of local seed density and in its fine-scale genetic composition (García et al., 2007a,b). Even in highly diversified plant-frugivore interactions, involving many species with dozens of interactions, only a few specific frugivores may contribute a major fraction of the long-distance dispersal events, or may contribute disproportionately to the seed rain in hot-spots for successful recruitment (Jordano and Schupp, 2000; Fragoso et al., 2003). Long-distance dispersal events, both within and between populations, are probably more frequent than previously thought for endozoochorous species, but it is not clear how they contribute to patterns of colonization and gene flow. Therefore, their pervasive implications for the maintenance of fragmented populations and for the demographic and genetic make-up of plant populations in complex landscapes remain poorly understood.

There are two key questions in seed dispersal studies:

1. Which frugivore species contribute dispersed seed where?

2. Which source trees contribute dispersed seed where?

These are very complex issues, especially for dispersal systems where the diversity of frugivore species is high. Answering the first question requires precise frugivore tracking; answering the second question requires precise seed tracking. The main problem is that tracking methods lose precision when the tracking time or the tracking distance increase, thus severely compromising our precision in assessing LDD events (Nathan et al., 2003). These are inherent problems for any of the methods used to date to assess dispersal distances.

The main components of seed shadow analysis are outlined in Table 10.1. From the perspective of the demographic and genetic effects of the frugivores, there are three main components of seed shadows: distance, density and location relative to the immediate neighbourhood (e.g. of conspecific trees). Let us consider the perspective of a dispersed seed just after successful dispersal to a microsite (target site) on the forest floor. 
Table 10.1. The main components of a seed shadow in relation to distance, density, and neighbourhood aspects and from the perspectives of the source tree for dispersed seeds and from the target microsite where seeds are delivered. Consider a dispersed seed in the seed shadow and its density, distance, and neighbourhood characteristics.

\begin{tabular}{|c|c|c|}
\hline Component & Source tree perspective & Target site perspective \\
\hline Distance & Distance from source tree & $\begin{array}{l}\text { Distance from source tree and to } \\
\text { nearest conspecific }\end{array}$ \\
\hline Density & $\begin{array}{l}\text { Density of dispersed seeds } \\
\text { from the source tree at } \\
\text { target site }\end{array}$ & $\begin{array}{l}\text { Density of dispersed seeds in } \\
\text { target site } \\
\text { Density of trees in target site } \\
\text { neighbourhood }\end{array}$ \\
\hline Neighbourhood & $\begin{array}{l}\text { Overlap of seed shadow } \\
\text { with conspecifics } \\
\text { Genetic relatedness of source } \\
\text { trees contributing seed }\end{array}$ & $\begin{array}{l}\text { Genetic relatedness of } \\
\text { dispersed seeds in target site } \\
\text { Diversity of source trees } \\
\text { contributing seed }\end{array}$ \\
\hline
\end{tabular}

Traditional seed shadow analysis has considered the relevance of both distance and density effects relative to the source trees (Janzen, 1970): the distance the seed has moved from the source tree, and the density of seeds from that source tree at the target point. By taking a 'target site perspective', it is also relevant to consider the distance to other conspecific trees, and the density of both dispersed seeds (from other conspecific trees) and adult trees in the neighbourhood of the target site (Table 10.1). The 'target site perspective' emphasizes that survival conditions for dispersed seeds might vary enormously in a context-dependent way (see Schupp, Chapter 20, this volume) because the 'safeness' of a site depends not only on distance to the seed source, but also on the environmental context of the target site.

\section{Direct observation}

Direct observations of frugivores while foraging for fruits reveal patterns of potential seed delivery, although it is extremely difficult to estimate actual dispersal from direct observation (Gómez, 2003; Jordano and Schupp, 2000; Wenny and Levey, 1998). In addition, reliable estimation of long dispersal distances, even within a local population, is generally not feasible. Direct observation of beween-population seed dispersal via LDD events, involving actual seed immigration, is almost impossible. Direct observation methods have been successfully used in acorn-caching jays (Garrulus glandarius) (Gómez, 2003), enabling the reliable measurement of long-distance flights ending in successful caching. However, the limitation persists for direct observation of acorn transfer between populations, especially between distant patches (J.M. Gómez, 2005, personal communication). For legitimate frugivores ingesting whole fruits there is the added complication of ingestion, retention time, and delayed delivery after gut mixing of fruits consumed during various feeding bouts (Holbrook and Smith, 2000; see 
Traveset et al., Chapter 4, this volume). The best approximations are obtained when watching habituated animals directly (Yumoto, 1999), using direct observations combined with non-intrusive methods (Mack and Druliner, 2003; Forget and Wenny, 2005; Levey et al., 2005) or with domestic species (Manzano and Malo, 2006).

Almost the same limitations arise when using radio-tracking techniques to infer seed-source tree distances and spatial relations. Despite a high reliability of the frugivore location at a given time interval, there is considerable uncertainty about the actual seed delivery event. One has to resort to functional digestion models and gut passage time estimates to infer the seed delivery events and estimate dispersal distances relative to potential source trees. This leads to a potential bias in the estimation of true seed-source tree distance patterns. Despite these potential limitations, radio-tracking studies have shown very detailed patterns of seed delivery by frugivores involving LDD events (Fleming, 1988; Sun et al., 1997; Holbrook and Smith, 2000; Westcott and Graham, 2000; Holbrook et al., 2002; Westcott et al., 2005). Direct observation or monitoring methods, if combined with seed rain monitoring (Wenny and Levey, 1998; Jordano and Schupp, 2000) will allow very robust estimates of who contributes seeds where, whereas the origin and distance components will be difficult to assess.

\section{Hypervariable DNA markers}

DNA-based methods to assess the parentage (maternity) of dispersed seeds have recently been developed, largely as an extension of seedling parentage analysis and pollen paternity analyses (Ennos, 2001). Briefly, parentage analysis uses genetic marker data from adults and established seedlings in a population to infer the male and female parents of focal offspring. Maternity analysis represents a subset of parentage techniques by assuming that established offspring (e.g. seedlings) derive from random mating of maternal trees with a homogeneous outcross pollen pool (Ennos, 2001; Jones and Ardren, 2003). Therefore, these techniques allow an inference based on exclusion probabilities and likelihood estimation. However, by taking advantage of tissue of maternal origin still attached to the dispersed seed in some way (e.g. endocarp tissue, ancillary structures of winged seeds like pappus, wings, acorn pericarp, etc), assignment of the most likely source tree can be inferred directly and robustly (Godoy and Jordano, 2001; Jordano and Godoy, 2002; Ziegenhagen et al., 2003; Grivet et al., 2005; Jones et al., 2005; García et al., 2007a). In contrast to direct observation methods, parentage and maternity analysis of dispersed seeds works from the dispersed seed backwards to infer the origin.

Methods not relying on the genotyping of seed maternal tissue use established seedlings and indirect inference methods to assign maternity and, eventually, paternity (Alvarez-Buylla et al., 1996; Schnabel et al., 1998; Bacles et al., 2006; Hardesty et al., 2006; Sato et al., 2006). While these 
methods provide direct evidences of LDD events (see Hardesty, Chapter 12 , this volume) they might underestimate the actual frequency of LDD events mediated by frugivore movements, since they measure established seedlings, i.e. realized, or effective, dispersal (Cain et al., 2000) after the effect of post-delivery mortality factors (failure to germinate, consumption by seed predators). Therefore, actual immigration rates of seeds can be underestimated and differentiation between the seed recruitment curve and the seedling recruitment curve cannot be accomplished (Jones et al., 2005). However, these methods can be very informative about the patterns of realized dispersal - a key ingredient of seed shadow analysis.

Genotyping methods based on maternal tissue can provide a useful tool when combined with direct watches of frugivores and seed rain analysis with seed traps. Based on direct assignment, they can overcome some of the limitations of likelihood-based assignment techniques (Jones and Ardren, 2003). By relying on sampling of dispersed seed, their main limitation would be the identification of the dispersal agent. Below I summarize a case study with Prunus mahaleb (Rosaceae) and discuss potential shortcomings of direct maternity estimation based on hypervariable DNA markers and endocarp tissues.

\section{Model fitting to dispersal data}

Valuable efforts in the last decade have been directed to model dispersal events, given the serious difficulties involved in empirically estimating dispersal distances. The two alternative approaches to dispersal modelling have been mechanistic and phenomenological (Nathan and Muller-Landau, 2000). Mechanistic models adopt a basic 'source tree' perspective and attempt to infer seed dispersal patterns from characteristics of the dispersers. They have been extensively used for anemochorous and abiotically dispersed species (Nathan et al., 2003). Phenomenological models implicitly adopt a 'target site' perspective, aiming at reconstructing the seed dispersal curve given seed-trap data and locations of potential source trees (Sato and Hiura, 1998; Clark et al., 1999). Given a pattern of dispersed seeds (e.g. derived from seed-trap sampling), inverse modelling is used to find out the best fit to a dispersal function that adequately reproduces the pattern, given the characteristics of the trees in the population (location, fecundity, etc). Additionally, these models can also take a source-tree perspective by assuming hypothetical distributions to represent the shape and scale of dispersal curves. Examples would be studies which estimate spread using different dispersal functions in the absence of any arrival data.

Invariably, the main difficulty with modelling attempts has been fitting the 'fat tail' of the dispersal curve: obtaining robust fits for the LDD events (Cain et al., 2000; Nathan, 2006), even for abiotically dispersed species for which the models can be adequately parametrized. The situation with mechanistic models for animal-dispersed species is similar, although there are very few examples available (Westcott and Graham, 2000; Westcott et al., 
2005; see Dennis and Westcott, Chapter 9, this volume). Recent approaches to modelling frugivore-mediated dispersal from a mechanistic perspective have successfully incorporated movement patterns that depend on frugivore foraging behaviour and habitat preferences, combined with gut passage time dynamics (Morales and Carlo, 2006). Basically, they model the seed dispersal kernels as a function of frugivore movement and seed passage times after regurgitation or defecation (Murray, 1988). Assuming that, after consuming fruit, animals perform a random walk, it is possible to approximate movement with a diffusion equation (Morales and Carlo, 2006). The solution of the diffusion equation is then combined with a probability density function for gut-passage time for seeds in order to solve for the distances at which they would be deposited. However, simple diffusion may be a poor approximation for frugivore movements (Holbrook et al., 2002; Westcott et al., 2005) that frequently entail more complex patterns than a pure random walk (Boyer et al., 2004). Furthermore, as stated by Morales and Carlo (2006), the spatial pattern of plant distribution will feed back into the characteristics of seed dispersal kernels via its effects on frugivore movements.

\section{A case study}

\section{An account of Prunus mahaleb dispersal}

Our protocols for the genetic evaluation of seed dispersal in Prunus mahaleb combine seed sampling in the field by means of seed traps that passively capture seeds dispersed (regurgitated or defecated) by frugivores, genetic analysis of adult trees and seed endocarps, and direct observations of frugivores at feeding trees. This enables us to identify uniquely the source tree for a dispersed seed and to infer the contribution of different species of frugivore to the seed rain in different microhabitats (Godoy and Jordano, 2001; Jordano and Godoy, 2002). Other applications of this approach can be found in recent work by Ziegenhagen et al. (2003), Grivet et al. (2005) and Jones et al. (2005).

Prunus mahaleb is a rosaceous tree that in south-eastern Spanish populations is gynodioecious, with some individuals producing hermaphrodite flowers and others with androsterile flowers, which behave as functional females. In the southern Iberian Peninsula, this species $f$ owers between mid-May and mid-June at high elevations (over $1300 \mathrm{~m}$ ), and insects, mainly bees (Hymenoptera: Andrenidae, Apidae) and flies (Diptera: Calliphoridae, Syrphidae), act as pollen vectors. Prunus mahaleb produces fleshy fruits (drupaceous) with one seed per fruit. In late July fl eshy fruits are produced and consumed by frugivorous animals that disperse the seeds until late August or early September.

Our nine local $P$. mahaleb populations are located in Parque Natural de las Sierras de Cazorla, Segura y Las Villas (Jaén province, south-east Spain). In this area $P$. mahaleb naturally occurs as isolated small (10 trees) to 
medium-sized (150 trees) distinct populations. A few populations might reach approx. 3000 trees. These populations are naturally isolated from each other and occupy approximately $150 \mathrm{~km}^{2}$. The main study population was located in Nava de las Correhuelas (NCH, hereafter), at $1615 \mathrm{~m}$ elevation. Detailed descriptions of the area and general methods can be found in Jordano and Schupp (2000) and references therein. The site is dominated by grasslands with scattered patches of deciduous vegetation, gravelly soil or rock outcrops covered by shrubs or small isolated trees. The rocky slopes are dominated by open pine forest (Pinus nigra subsp. salzmannii, Pinaceae) and juniper (Juniperus communis, Cupressaceae).

Frugivorous birds and mammals visiting $P$. mahaleb trees in Spanish populations usually behave as legitimate seed dispersers, swallowing the fruits whole and defecating and/or regurgitating the seeds, usually after leaving the tree. Most seed rain of $P$. mahaleb in the study areas is contributed by frugivorous birds. Seed rain and the resulting recruitment pattern of seedlings and saplings are highly patchy, and largely restricted to microhabitats beneath woody cover in the vicinity of fruiting trees (Jordano and Schupp, 2000).

Our general approach to assess seed dispersal distances and spatial patterns is to compare the multilocus genotype of the endocarp of dispersed seeds (either defecated or regurgitated by frugivorous animals) with those of potential maternal source trees in the population. Both hermaphrodite and female trees can act as seed sources in the study population, while only hermaphrodites act as pollen donors. The sex ratio is $<1: 1$ for the two gender types in this population. The endocarp is a maternal tissue (2n) with an identical genotype to the mother tree and in Prunus is derived from the carpellar wall. Thus, a full matching of the multilocus genotypes of a dispersed seed and a maternal tree unequivocally identifies the tree as the source for the dispersed seed, enabling a direct estimation of the dispersal distance (Godoy and Jordano, 2001).

\section{Source tree identification}

For this study of seed dispersal, a total of 180 adult trees and 95 dispersed seed endocarps were initially genotyped by Godoy and Jordano (2001). We later expanded this sample to include 263 trees from NCH (of which we used only the 196 reproductives in 2003; García et al., 2005, 2007a,b) and 557 endocarps, and this is currently our main analysis data set. We have also genotyped trees from another eight populations in addition to $\mathrm{NCH}$, totalling 472 trees.

Each adult tree in the NCH population showed a unique multilocus genotype. The source tree for individual dispersed seeds was identified by comparing the endocarp multilocus genotype with the complete set of genotypes of reproductive trees in the population. To assign the source tree for each dispersed seed we carried out an identity check by matching the multilocus genotype of the endocarp at nine microsatellite loci with those of 
the adult trees; for the adult trees we assessed 11 SSR loci. We used the full seed sample from the $1996(n=95)$ and $1997(n=462)$ cohorts (Table 10.2). We dropped $n=27$ endocarps due to amplification failure for $>2$ loci. We used CERVUS (Marshall et al., 1998) and GIMLET (Valière, 2002) to identify the mismatches and multiple-matches among endocarps and putative source trees. For each sampled seed, the adult individual having a genotype matching the seed endocarp genotype was assigned as the mother tree. In a few cases ( $n=27$ endocarps), we failed to find evidence that an NCH tree was the mother source, but they had $\leq 2$ loci missing and thus we were unable to assign them as immigrant seeds in $\mathrm{NCH}$ and so we considered them to be reassigned $\mathrm{NCH}$ seeds. Of the endocarps which could be assigned to $\mathrm{NCH}$ trees, all but two were assigned to a single tree; 97 endocarps were not assignable to any tree in $\mathrm{NCH}$ and we inferred that they were immigrant seeds dispersed from fruiting trees in other populations. We thus had 437 endocarps assigned to the NCH study population. The two seeds with double matching had failed amplifications for two loci and resulted in ambiguous matching (i.e. with two putative maternal trees). We assigned the seeds to the tree nearest to the sampling location, due to the fact that this procedure would minimize the estimation errors of dispersal distances when these are distributed with high skew. Incorrect assignment of a rare longdistance event would seriously bias the estimate of the dispersal function. Significant matches between endocarp and adult genotypes were found by testing a hypothesis of identity $\left(r_{1}=1, r_{\mathrm{m}}=1\right)$ in all possible pairwise comparisons between endocarps and adult trees and obtaining significance estimates by a jackknife resampling method (Queller and Goodnight, 1989).

\section{Problems with SSR genotyping}

There can be some problems at the genotyping and assignment stages when working with seed endocarps, and several of them are frequently encountered in maternity analyses.

Table 10.2. Estimates of the frequency (\%) of within- and betweenpopulation dispersal events of pollen and seeds in Prunus mahaleb. Pollen dispersal was inferred from paternity analysis of progeny samples taken from maternal tree canopies before consumption by frugivores. Seed dispersal events were inferred from seed progeny sampled in seed traps after dispersal by frugivores. $n=200$ seeds for pollen dispersal analysis (1997 cohort); $n=557$ seeds for seed dispersal analysis ( $n=95$ seeds for the 1996 cohort; $n=462,1997$ cohort). See Godoy and Jordano (2001), García et al. (2005, 2007a,b), Jordano et al. (2007).

\begin{tabular}{lcc}
\hline Stage & Within population & Between populations \\
\hline Pollen & 90.50 & 9.50 \\
Seeds & 79.66 & 20.34 \\
\hline
\end{tabular}


- Stutter bands are fairly common when analysing microstellites, especially with dinucleotide repeats. We have observed them in amplifications from endocarp extracts but also, to the same extent, with leaf extracts and with all the other species we have worked on. Alleles were assigned to the largest, and most abundant, fragment. Heterozygotes for two close alleles were recognized by the shorter allele showing a higher intensity than the larger (the intensity of the band being the sum of the short allele and the stutter bands of the long allele). This results in a characteristic pattern clearly distinguishable from the homozygote pattern, in which the intensity of the bands decreases progressively with size. This is described by Hoelzel (1998).

- Null alleles are alleles that do not amplify, probably because of a mismatch in one of the primers used. They can be suspected if a heterozygote deficit is detected only in some of the loci; they are frequent in parentage analysis (Björklund, 2005). If null alleles are present, they should appear both in leaf and endocarp extracts. Therefore, we think it unlikely that they would affect the identity checks between seeds and trees: a seed showing a false homozygote will match a tree showing the same pattern. However, this will affect and limit the resolution power and bias the significance values for assignments (Jones and Ardren, 2003) (see below). We did not discount the possibility that null alleles were present in our samples, but we did not detect them. We observed a heterozygote deficit in most loci resulting from high inbreeding in the study population due to frequent selfing. We have not detected null alleles in paternity analyses (embryo genotyping), where we used progeny with known paternal and maternal trees, these being obtained from hand pollinations.

- Allelic dropout can also be a serious problem for the assignment of seeds to mother trees. It occurs when one of the alleles in a heterozygote is not amplified stochastically when using limited template DNA, as can be the case when endocarp extracts are used. In the controlled comparisons we made between leaf and endocarp genotypes, allelic dropout was not observed. However, this can be a potential problem if the DNA yield or quality is limiting. Repeating the amplification of homozygote loci several times (Taberlet, 1996) and accurately determining the concentration of DNA and excluding those samples in the limiting range where allelic dropout can occur (Morin et $a l ., 2001)$ are two strategies to deal with the problem at the genotyping stage. At the analysis level, any exclusion of identity between a seed and a potential mother tree based on only 1 or 2 loci mismatching (i.e. $<10 \%$ of the seeds in our sample) was rechecked.

\section{Seed dispersal distances}

Seed dispersal distances can readily be determined with the direct assignment method based on endocarp genotypes. For P. mahaleb we have 
already reported distances with a reduced sample of seeds (Godoy and Jordano, 2001; Jordano and Godoy, 2002). The pattern with a more extensive sample is quite similar, revealing highly limited dispersal in terms of distance, with most seeds dispersed " $\$ 50 \mathrm{~m}$ from the source tree (Fig. 10.1) (Jordano and Godoy, 2002; García et al., 2007a). Median dispersal distance (including only within-population events) was $32.0 \mathrm{~m}$ with quartiles $Q_{25}=10.5 \mathrm{~m}$ and $Q_{75}=100.3 \mathrm{~m}$ ( $n=437$ seeds). It is worth noting that $Q_{99.5}=1025.0 \mathrm{~m}$, indicating that LDD events within a population can occur with low frequency; thus, $3.5 \%$ of the dispersal events had a mean dispersal distance of $793.8 \mathrm{~m}$. The resulting seed dispersal curve agrees well with the intra-population distances estimated from direct watches of birds while foraging at feeding trees (Jordano and Schupp, 2000 ) but, as expected, gives a much more precise and robust estimation of the LDD events. The dispersal curve was markedly leptokurtic (kurtosis $=$ 16.68), with mode at $\leq 50 \mathrm{~m}$ and maximum at $1025 \mathrm{~m}$. Note that in Figure 10.1, dispersal events within $1200 \mathrm{~m}$ distance indicate within-population dispersal: seeds dispersed from trees growing in the $\mathrm{NCH}$ population. Only a small fraction of the dispersal events recorded correspond to immigrant seeds dispersed from other populations, and these LDD events are represented in Figure 10.1 by the bars beyond $1200 \mathrm{~m}$.

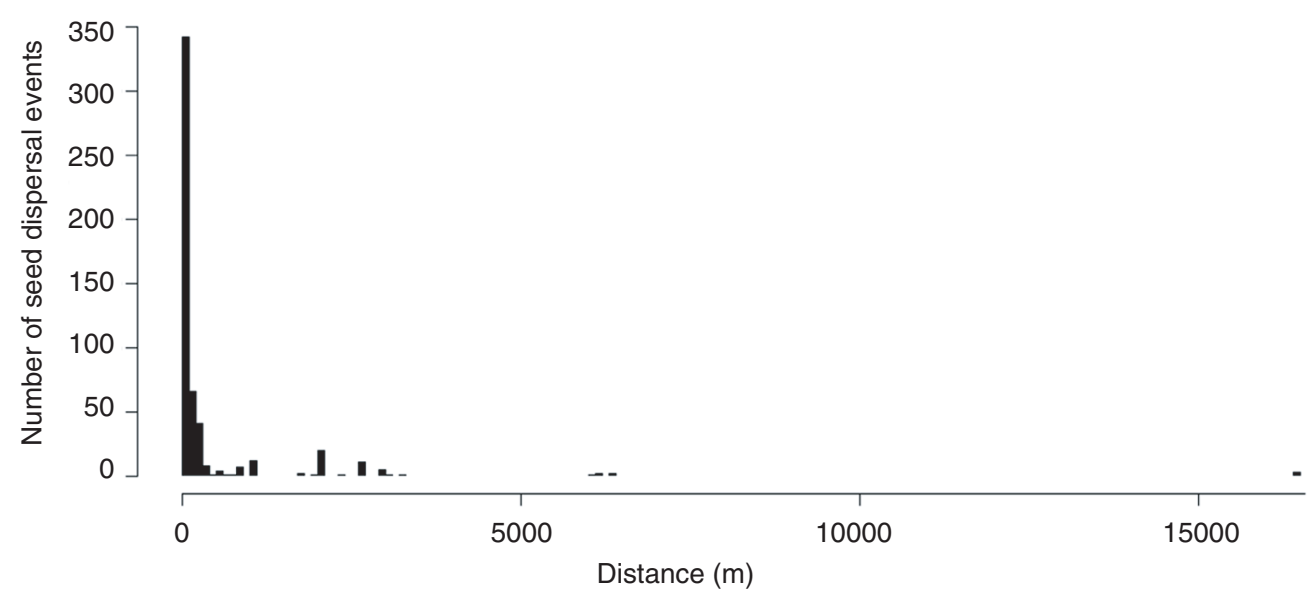

Fig. 10.1. Distance patterns in the seed rain of Prunus mahaleb ( $n=558$ seeds): the frequency distribution of dispersal distances in the study population including the long-distance between-population dispersal estimates. The dispersal curve combines results of seed dispersal distances from trees growing in the population (direct assignment) with distance estimates for immigrant seeds (assignment to the most likely source population). The abcissa shows the actual distance values between the location of a dispersed seed (defecated or regurgitated by a frugivore) and its maternal tree (log-scale, actual distance $+1 \mathrm{~m}$; distance $0 \mathrm{~m}$ includes seeds falling beneath the canopy of the mother tree) or source population. The dispersal distances within the population (i.e. not including seeds originating from other populations), reach $1200 \mathrm{~m}$; those corresponding to immigrant seeds are indicated by the bars beyond this distance ( $n=97$ seeds with provenance assigned to other populations). 
We were recently able to estimate the relative contribution of different frugivore types to the dispersal curve, estimating the total dispersal kernel (TDK; see Nathan, Chapter 11, this volume) (Jordano et al. 2007). We analysed endocarps from faecal samples, pellets or remains that we identified as having been dispersed by different frugivore groups including small- and medium-sized birds (warblers, redstarts, thrushes, crows), and carnivorous mammals (red fox, stone marten and badger). This way, were able to assign dispersal distances to seeds that we know had been dispersed by these frugivores. Whereas small-sized birds were by far the main seed dispersers up to $250 \mathrm{~m}$, larger frugivores dispersed most of the seeds to distances up to $1100 \mathrm{~m}$, indicating that seed dispersal within the population can be extensive but highly structured spatially. Medium-sized birds (Turdus viscivorus, Turdidae; Corvus corone, Corvidae; and, most likely, Columba palumbus, Columbidae) contributed most of the LDD events within the population by dispersing seeds mostly beyond $100 \mathrm{~m}$; but they also contributed to short dispersal distances (especially T. viscivorus) (Jordano et al., 2007). Seed dispersal distances by carnivorous mammals ranged from $0 \mathrm{~m}$ (i.e. under the source tree canopy) up to $990 \mathrm{~m}$, with a peak at 650-700 $\mathrm{m}$ (Jordano et al., 2007). These distance intervals obviously correspond to within-population dispersal events (seeds consumed in trees growing in the study population); but seeds can be moved longer distances when being dispersed from other populations.

We tracked the origin of immigrant seeds dispersed from other populations by using assignment methods based on Bayesian inference techniques (Wilson and Rannala, 2003). Briefly, endocarps not assigned to any tree within the focal $\mathrm{NCH}$ population can be assigned to one of the eight sampled populations based on the allelic frequencies of the trees growing in them. Given that an endocarp sampled in a seed trap in the focal population $(\mathrm{NCH})$ does not match any source tree in the population, we basically ask what is the likelihood that the seed came from any of the eight populations (located between 1.5 and $17 \mathrm{~km}$ away from $\mathrm{NCH}$ ), given its multilocus genotype and the allelic frequencies of each population. We performed this test with the non-NCH seeds and included the distance interval between NCH and each of these populations in Figure 10.1, thus obtaining the 'extended' seed dispersal curve that includes all the LDD events recorded in the seed sample studied. This reveals an unexpectedly high frequency, up to $20.34 \%$ (Table 10.1), of immigrant seeds (Godoy and Jordano, 2001; Jordano and Godoy, 2002; P. Jordano and J. Godoy, 2006, unpublished results; García et al., 2007a; Jordano et al., 2007).

When including the distance estimates for immigrant seeds, the dispersal curve extends to median $=62.0 \mathrm{~m}$ with quartiles $Q_{25}=13.5 \mathrm{~m}$ and $Q_{75}=274.0 \mathrm{~m}$ ( $n=557$ seeds, the whole seed sample), with a maximum of $16,400 \mathrm{~m}$ for a LDD event recorded for the Torcal Llano population, $\geqslant 16 \mathrm{~km}$ from $\mathrm{NCH}$. We have evidence that most immigrant seeds in the study population are dispersed by carnivorous mammals, including badger (Meles meles, Mustelidae), stone marten (Martes foina, Mustelidae) and red fox (Vulpes vulpes, Canidae) (Jordano et al., 2007). 
Their weighted contribution to the immigrant seed pool (considering both the fraction of immigrant seeds in their samples and their quantitative seed removal) was $66.9 \%$, while frugivorous birds accounted for the remaining $33.1 \%$. When considering each dispersal vector separately, we observed that $74.2 \%$ of the seeds dispersed by mammals were imported from outside the population, whereas $21.9 \%$ of the seeds dispersed by birds came from other populations. While these are relative figures, it must be noted that, quantitatively, frugivorous birds disperse a considerably larger amount of P. mahaleb seeds than mammals do (Herrera and Jordano, 1981; Jordano and Schupp, 2000). Whereas small-sized birds dispersed seeds mainly beneath the canopies of $P$. mahaleb and other fleshy-fruited trees or shrubs, mammals deposited dispersed seeds preferentially in open sites (rocky soils and open ground with little woody vegetation or grass cover). Mediumsized birds dispersed seeds mainly to open areas $(C$. corone) and beneath pine trees (T. viscivorus).

A number of studies have reported very long dispersal events mediated by frugivores, ranging between hundreds of metres (Wenny and Levey, 1998; Gómez, 2003; Forget and Wenny, 2005) to thousands of metres (Fleming, 1988; Mack, 1995; Yumoto, 1999; Levey et al., 2005), to kilometres (Holbrook and Smith, 2000; Westcott and Graham, 2000; Oddou-Muratorio et al., 2001; Holbrook et al., 2002; Fragoso et al., 2003; Westcott et al., 2005; Jordano et al., 2007). In addition, reported dispersal distances for established seedlings from animal-dispersed seeds range up to kilometres (Hardesty et al., 2006). Despite this increase in our understanding of LDD events, we still lack a clear picture of the actual frequency associated with events in different parts of the dispersal curve and, most especially, those at the tail of the distribution (Cain et al., 2000; Nathan, 2006). We still lack detailed data on the frequency of immigrant seeds dispersed from other populations into a given population or fragment and on how different frugivore species contribute to this. We especially need a tighter integration of ecological and molecular DNAbased methods to be able to assess dispersal patterns in high-density situations (Grivet et al., 2005; Jones et al., 2005; Hardesty et al., 2006) and in complex systems where the total dispersal kernel (see Nathan, Chapter 11, this volume) can have very distinct components. However, it is becoming more and more evident that frugivores potentially have a dramatic influence on LDD events and patterns of gene flow via seed dispersal (Godoy and Jordano, 2001; Oddou-Muratorio et al., 2001; Webb and Peart, 2001; García et al., 2005; Bacles et al., 2006; Hardesty et al., 2006; Jordano et al., 2007) compared with pollinators; a view not traditionally supported in the literature (Ennos, 2001). Our data with $P$. mahaleb suggest that seed-mediated gene flow probably doubles pollenmediated gene flow in the studied populations, and this scenario might well be generalizable to other species of tree and shrub with animal pollinators and dispersers (Oddou-Muratorio et al., 2001; García et al., 2007a,b). Moreover, these data are compatible with recent interpretations of old paradoxes about LDD in biogeographic scenarios, like the 
postglacial colonization of Northern continental areas in Europe and North America (Nathan, 2006). The data on LDD of P. mahaleb also emphasize a central role of frugivores in the connectivity of fragmented populations via LDD events (see also Levey et al., 2005), a central issue in conservation of altered habitats. Given that plant-frugivore interactions are typically of low specificity and high diversity, involving many species of disperser for a given species of plant, this evidence shows that probably only a small subset of the frugivore coterie has a disproportionately high contribution to LDD events (Jordano et al., 2007; see Dennis and Westcott, Chapter 9, this volume; see Nathan, Chapter 11, this volume).

\section{Spatial heterogeneity: 2-D patterns and the seed shadow}

Seed dispersal curves emphasize a distance component of seed dispersal. The frequency distribution of dispersal distances is usually represented as a function of directional distance (Nathan and Muller-Landau, 2000). But it is clear that seed dispersal is a 2-D process (sometimes a 3-D process, when, e.g. epiphytes and mistletoes are involved), as emphasized by Janzen (1970) in his seminal paper. Two-dimensional patterns in seed shadows originate chiefly from the interaction of three components; namely, the location of fruiting trees, frugivores' foraging behaviour and habitat preferences, and the major elements of complex landscapes (e.g. forest edges, rock outcrops, ravines, gaps, etc.). Recent findings from animal-movement analysis have revealed that characteristic 2-D patterns in frugivore movement may markedly deviate from purely random walks (Fig. 10.2) (Viswanathan et al., 2002) and can potentially influence the structure of seed shadows (Morales and Carlo, 2006).

While purely Brownian random walks are built up from relatively similar displacements or steps, with a characteristic step length $\ell$ and a random turning angle, movements and search patterns of biological organisms typically follow a particular type of random walk termed a Lévy walk or, more generally, a Lévy flight pattern (Fig. 10.2) (Viswanathan et al., 2002; Bartumeus, 2005). In a random walk there is a characteristic scale of displacement, defined by a mean step length $\ell$; most movements imply a similar distance, the average displacement $\ell$, and there are no extremely long steps (i.e. LDD events do not exist). The probability of observing a movement of a given step length $\ell_{j}$ is given by a Gaussian distribution. In contrast, actual movement patterns of foraging animals have been found to be better represented by Lévy walks (Viswanathan et al., 2002), where the probability of observing a path of length $\ell$ is given by:

$$
\mathrm{P}\left(\ell_{j}\right) \sim \ell_{j}^{-u}
$$

Thus, the probability of finding a given step length is best described as a function that follows a power-law distribution in which the frequency of LDD events decreases as a negative power function of their length. We find many small dispersal distances but we can also find extremely long 

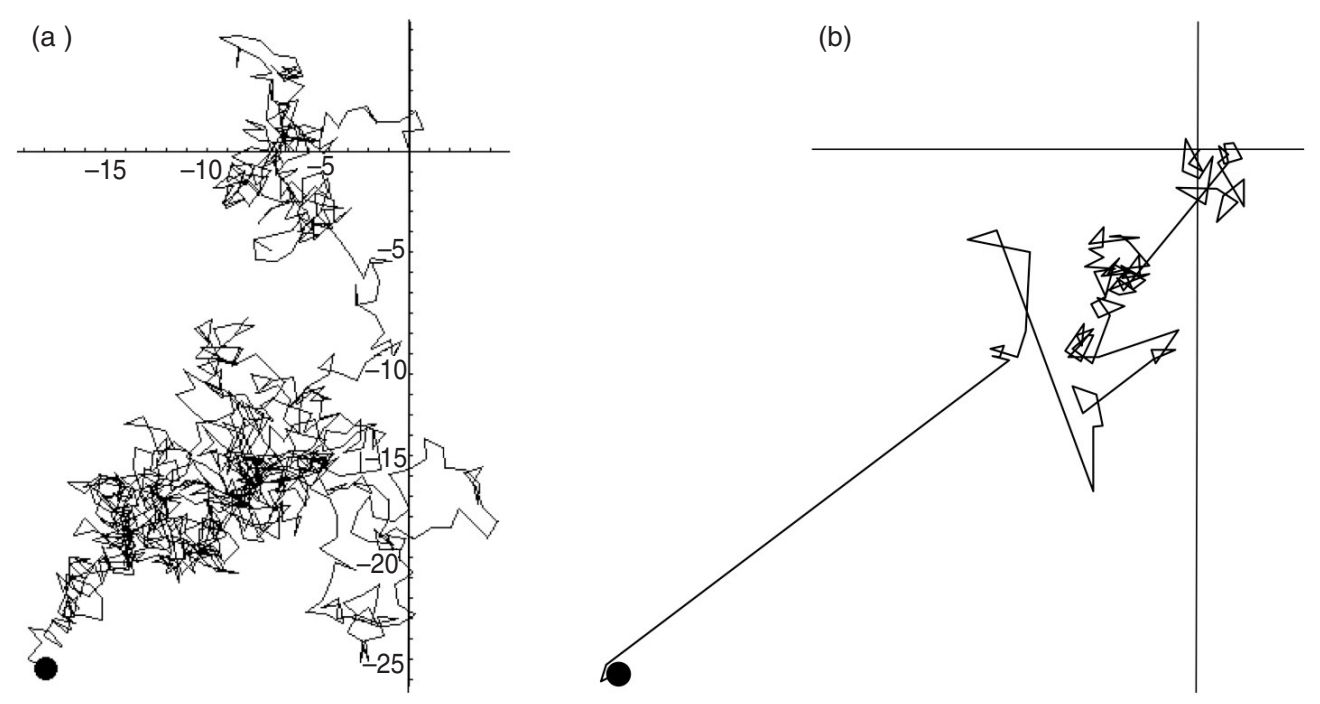

Fig. 10.2. Two simulated trajectories of movement patterns by animal frugivores: (a) a random walk pattern ( $n=5000$ trajectories); (b) a Lévy walk pattern ( $n=190$ trajectories). Axes are reference lines.

dispersal events, which occur with very low probability. In a purely Brownian motion model, these long steps simply do not exist. The parameter $\mu$ is the exponent that fully characterizes such a distribution and can be determined from empirical data. In contrast with random walks, Lévy walks have spatial scale invariance in step lengths ${ }^{2}$ and in the duration of intervals between steps (Viswanathan et al., 2002). As a consequence, Lévy walks do not have a characteristic step scale and steps of extremely long length can be recorded (Fig. 10.2) (Ramos-Fernandez et al., 2004; Bartumeus, 2005). Ramos-Fernandez et al. (2004) have recently reported foraging patterns of Ateles geoffroyi (Cebidae) in Yucatán best described as Lévy walks. In addition, some of the results reported by frugivore radio-tracking studies (Westcott and Graham, 2000; Holbrook et al., 2002; Fragoso et al., 2003; Westcott et al., 2005; see Dennis and Westcott, Chapter 9, this volume) suggest similar patterns.

The seed dispersal curve of $P$. mahaleb is extremely leptokurtic and has a characteristic Lévy walk pattern. That is, if we represent all the steps moved by the seeds dispersed from a given tree, or from the tree population as a whole (derived from Fig. 10.1), we should obtain a pattern of step lengths markedly deviating from a Brownian random walk and approaching a Lévy walk (Fig. 10.3a). The dispersal curve for P. mahaleb seeds deviates markedly from what would be expected if the frugivores were simply following the location of the fruiting trees while foraging: it has a fatter tail, with a higher probability of steps (dispersal distances) beyond $>100 \mathrm{~m}$. Moreover, the associated exponent $\mu=1.67$ is very close to that expected for a Lévy walk. In comparison, the tail of the distribution of inter-tree distances (Fig. 10.3a) drops suddenly at shorter distances 

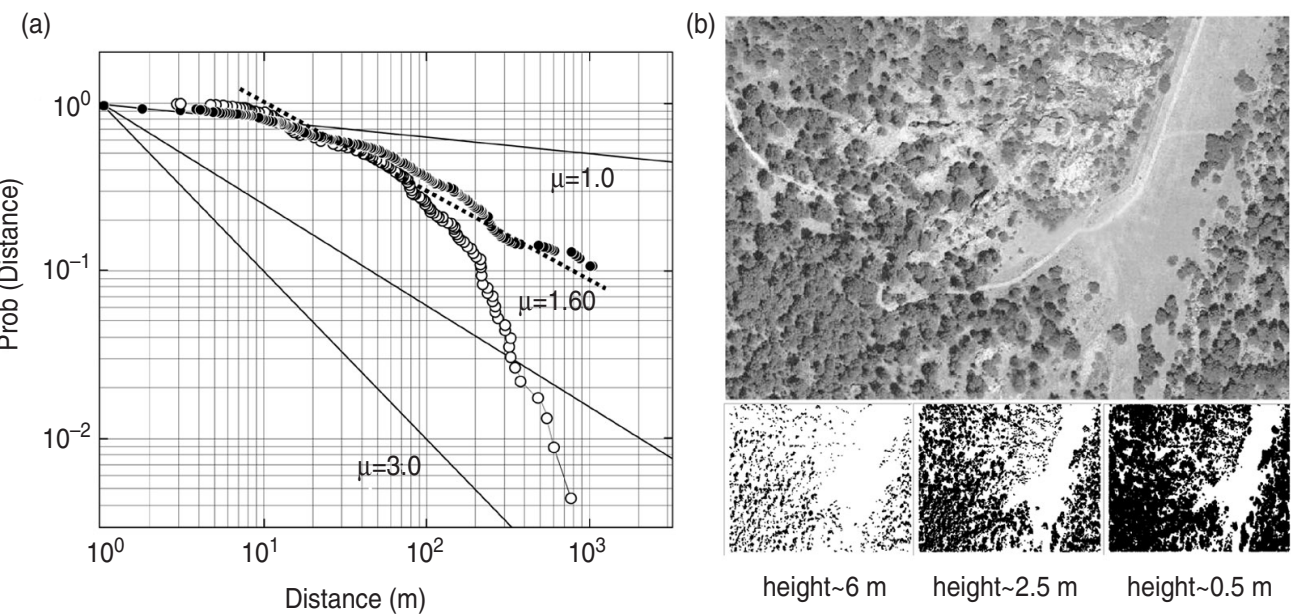

Fig. 10.3. The distribution of seed dispersal distances in relation to habitat characteristics and landscape heterogeneity. (a) Cumulative frequency distributions for dispersal distances. Filled dots, the observed distribution of seed dispersal distances; blank dots, the distance distribution that would be expected under a pattern of frugivore foraging that follows exactly the location of fruiting trees, based on tree nearest-neighbour distances. The slopes for $\mu=1.0-3.0$, drawn for reference, indicate the expected slopes for three different movement patterns, with $\mu=1.60$ approaching a Lévy walk. Note that the slope for seeds (dashed line) closely approaches the expected pattern with Lévy walk movement $(\mu=1.67)$ and markedly deviates from the slope of a random walk with purely Brownian motion $(\mu=3.00)$. (b) Aerial photograph of the study area and three slices of plant cover at three different heights $(0.5,2.5$ and $6 \mathrm{~m})$, illustrating the different patchiness and heterogeneity of plant cover defining major landscape elements such as the pine forest edge, rocky slopes (light grey areas) and open grassland with no shrubs (large grey area).

$(100 \mathrm{~m})$, reflecting the aggregation pattern of the trees in the area. This implies that the dispersal distances contributed by frugivores closely map the spacing patterns of fruiting trees, but only up to a certain distance $(\leq 100 \mathrm{~m}$; Fig. 10.3a). Beyond this, frugivores are probably responding to other major landscape elements (e.g. rock outcrops, forest edges, large patches of open grassland, etc) that cause the fat tail of the seed dispersal distribution, adding more frequent LDD events than expected from a Brownian random walk pattern generated by a tracking of the crops of the fruiting trees. For instance, the long flights performed by T. viscivorus (Jordano and Schupp, 2000) frequently face the pine forest edge, at distances $\geqslant 100 \mathrm{~m}$ of most $P$. mahaleb fruiting trees (Fig. 10.3b). If these medium-sized birds are selecting habitat with tall woody vegetation (e.g. pines $>6 \mathrm{~m}$ height), then they should be 'perceiving' a much more patchy landscape, and thus requiring longer flights, than for example, small warblers seeking vegetation cover $<0.5 \mathrm{~m}$ (Fig. 10.3b). In addition, these major landscape elements certainly have a lasting effect on non-random directionality patterns in the population-level seed dispersal, as reported recently for P. mahaleb (García et al., 2007b). 
Two-dimensional patterns in the $P$. mahaleb seed rain and the individual seed shadows, accurately tracked with DNA-based genotyping methods, thus reflect the complex effects of frugivore foraging, habitat preferences and heterogeneous landscapes. This situation is probably generalizable to other plant-frugivore interactions (Morales and Carlo, 2006) where the combined spatial dynamics of habitat use and digestion processes determine complex seed shadows (Jordano et al., 2007; see Dennis and Westcott, Chapter 9 this volume; see Nathan, Chapter 11, this volume). Much of this complexity can be adequately handled by mechanistic models (Nathan et al., 2002; Morales and Carlo, 2006) incorporating very simple rules (Viswanathan et al., 2002) that successfully recover the patterns revealed by molecular genetic techniques and direct watches of foraging animals (Jordano et al., 2007). However, a better integration of these different approaches is needed in future research.

\section{Perspectives: extending the seed shadow}

Recent advances in the study of seed dispersal by frugivores have dramatically increased our ability to assess seed shadows and to understand dissemination patterns mediated by animals. The most promising avenues for research in this area are clearly transdisciplinary in nature, requiring at least three main ingredients:

1. solid field data on how plant distribution influences disperser behaviour and movements

2. careful use of molecular tools and assignment/parentage analysis based on hypervariable DNA markers

3. robust modelling techniques that explicitly incorporate spatial heterogeneity.

This point has been repeatedly emphasized in recent reviews specific to each of these three components (Cain et al., 2000; Ennos, 2001; Jordano and Godoy, 2002; Wang and Smith, 2002; Jones and Ardren, 2003; Nathan et al., 2003; Forget and Wenny, 2005; Nathan, 2006; García, 2007). Adequate and viable means of synthesizing from among these approaches have been identified and are starting to be applied in current research.

Extending the seed shadow means increasing our understanding of the extremely rare, yet recurrent, LDD events that occur in nature (Nathan, 2006) and a better understanding of their reach, extent and frequency. Dispersal events have a pervasive influence on gene-flow patterns, genetic structuring, colonization ability, connectivity between fragmented areas, and cohesiveness in metapopulation scenarios. Despite the high intrinsic diversity of plant-frugivore interactions, only a few species, independently of their quantitative importance in fruit removal, might account for these rare events of far-reaching biological significance. Conversely, short-distance events are a major influence on local population recruitment and in the successful buildup of a large propagule source able to realize successful LDD. 


\section{Acknowledgements}

I am indebted to Manolo Carrión, Juan Luis García-Castaño, Jesús Rodríguez and, especially, Cristina García, Juan Miguel Arroyo and José A. Godoy for generous help with field and laboratory work and for making this study possible. Discussions with José A. Godoy, Cristina García, Juan Miguel Arroyo, Eugene W. Schupp, Janis Boettinger, Juan Luis GarcíaCastaño, Miguel Angel Fortuna and Jordi Bascompte were very helpful during all the stages of this manuscript and, over the years, have helped enormously to focus my research on Prunus genetics. I would like to thank the organizers of the Fourth International Symposium/Workshop on Frugivores and Seed Dispersal - Andrew Dennis, Ronda Green, Eugene W. Schupp and David Westcott - for their invitation to contribute to this volume and for their help and advice during the editing process. Comments by Cristina García, Alfredo Valido, Arndt Hampe and two anonymous reviewers helped to improve drafts of the manuscript. The study was supported by the Spanish Ministerio de Ciencia y Tecnología (BOS2000-1366-C02-01, REN2003-0273 and CGL2006-00373 to P.J.). Over the years, the Consejería de Medio Ambiente, Junta de Andalucía, provided generous facilities that made possible this study in the Sierra de Cazorla and authorized my work there.

\section{References}

Alcántara, J., Rey, P., Valera, F. and Sánchez-Lafuente, A. (2000) Factors shaping the seedfall pattern of a bird-dispersed plant. Ecology 81, 1937-1950.

Alvarez-Buylla, E., Chaos, A., Piñero, D. and Garay, A. (1996) Demographic genetics of a pioneer tropical tree species: patch dynamics, seed dispersal, and seed banks. Evolution 50, 1155-1166.

Bacles, C.F.E., Lowe, A.J. and Ennos, R.A. (2006) Effective seed dispersal across a fragmented landscape. Science 311, 628.

Bartumeus, F. (2005) Lévy processes in animal movement and dispersal. PhD thesis, University of Barcelona, Spain.

Björklund, M. (2005) A method for adjusting allele frequencies in the case of microsatellite allele drop-out. Molecular Ecology Notes 5, 676-679.

Boyer, D., Miramontes, O., Ramos-Fernandez, G., Mateos, J.L. and Cocho, G. (2004) Modeling the searching behaviour of social monkeys. Physica A 342, 329-335.

Bullock, J.M. and Clarke, R.T. (2000) Long-distance seed dispersal by wind: measuring and modelling the tail of the curve. Oecologia 124, 506-521.

Cain, M., Milligan, B. and Strand, A. (2000) Long-distance seed dispersal in plant populations. American Journal of Botany 87, 1217-1227.

Clark, C.J., Poulsen, J.R., Connor, E.F. and Parker, V.T. (2004) Fruiting trees as dispersal foci in a semi-deciduous tropical forest. Oecologia 139, 66-75.

Clark, J., Silman, M., Kern, R., Macklin, E. and Hilleris-Lambers, J. (1999) Seed dispersal near and far: patterns across temperate and tropical forests. Ecology 80, 1475-1494.

Ennos, R. (2001) Inferences about spatial processes in plant populations from the analysis of molecular markers. In: Silvertown, J. and Antonovics, J. (eds) Integrating Ecology and Evolution in a Spatial Context. Blackwell Science, London, pp. 45-72. 
Fleming, T. (1988) The Short-Tailed Fruit Bat. A Study in Plant-Animal Interactions. Chicago University Press, Chicago, IL.

Forget, P.M. and Wenny, D. (2005) How to elucidate seed fate? A review of methods used to study seed removal and secondary seed dispersal. In: Forget, P.M., Lambert, J.E., Hulme, P.E. and Vander Wall, S.B. (eds) Seed Fate: Predation, Dispersal and Seedling Establishment. CAB International, Wallingford, UK, pp. 379-393.

Fragoso, J.M.V., Silvius, K.M. and Correa, J.A. (2003) Long-distance seed dispersal by tapirs increases seed survival and aggregates tropical trees. Ecology 84, 1998-2006.

García, C. (2007) A unified framework for the study of dispersal in plants. Evolutionary Ecology 00, 000-000.

García, C., Arroyo, J.M., Godoy, J.A. and Jordano, P. (2005) Mating patterns, pollen dispersal, and the ecological maternal neighbourhood in a Prunus mahaleb population. Molecular Ecology 14, 1821-1830.

García, C., Jordano, P. and Godoy, J.A. (2007a) Spatial and maternal source tree correlations in a frugivore-generated seed rain. Journal of Ecology 00, 000-000.

García, C., Jordano, P. and Godoy, J.A. (2007b) Contemporary pollen and seed dispersal in a Prunus mahaleb population: patterns in distance and direction. Molecular Ecology 16, 000-000.

Godoy, J.A. and Jordano, P. (2001) Seed dispersal by animals: exact identification of source trees with endocarp DNA microsatellites. Molecular Ecology 10, 2275-2283.

Gómez, J.M. (2003) Spatial patterns in long-distance dispersal of Quercus ilex acorns by jays in a heterogeneous landscape. Ecography 26, 573-584.

Grivet, D., Smouse, P.E. and Sork, V.L. (2005) A novel approach to an old problem: tracking dispersed seeds. Molecular Ecology 14, 3585-3595.

Hardesty, B.D., Hubbell, S.P. and Bermingham, E. (2006) Genetic evidence of frequent longdistance recruitment in a vertebrate-dispersed tree. Ecology Letters 9, 516-525.

Harms, K.E., Wright, S.J., Calderón, O., Hernández, A. and Herre, E.A. (2000) Pervasive density-dependent recruitment enhances seedling diversity in a tropical forest. Nature 404, 493-495.

Herrera, C. and Jordano, P. (1981) Prunus mahaleb and birds: the high efficiency seed dispersal system of a temperate fruiting tree. Ecological Monographs 51, 203-221.

Hoelzel, A.R. (1998) A method for adjusting allele frequencies in the case of microsatellite allele drop-out. In: Hoelzel, A.R. (ed.) Molecular Genetic Analysis of Populations: A Practical Approach. Oxford University Press, Oxford, UK, pp. 234-245.

Holbrook, K.M. and Smith, T.B. (2000) Seed dispersal and movement patterns in two species of ceratogymna hornbills in a west African tropical lowland forest. Oecologia 125, 249-257.

Holbrook, K.M., Smith, T.B. and Hardesty, B.D. (2002) Implications of long-distance movements of frugivorous rain forest hornbills. Ecography 25, 745-749.

Houle, G. (1992) Spatial relationship between seed and seedling abundance and mortality in a deciduous forest of north-eastern North America. Journal of Ecology 80, 99-108.

Howe, H.F. and Kerckhove, G.A.V. (1980) Nutmeg dispersal by tropical birds. Science 210, 925-927.

Izhaki, I., Walton, P. and Safriel, U. (1991) Seed shadows generated by frugivorous birds in an eastern Mediterranean scrub. Journal of Ecology 79, 575-590.

Janzen, D. (1970) Herbivores and the number of tree species in tropical forests. American Naturalist 104, 501-528.

Jones, A.G. and Ardren, W.R. (2003) Methods of parentage analysis in natural populations. Molecular Ecology 12, 2511-2523.

Jones, A., Chen, J., Weng, G. and Hubbell, S. (2005) A genetic evaluation of seed dispersal in the neotropical tree, Jacaranda copaia (Bignoniaceae). American Naturalist 166, 543-555. 
Jordano, P. and Godoy, J. (2002) Frugivore-generated seed shadows: a landscape view of demographic and genetic effects. In: Levey, D., Silva, W. and Galetti, M. (eds) Frugivores and Seed Dispersal: Ecology, Evolution, and Conservation. CAB International, Wallingford, UK, pp. 305-321.

Jordano, P. and Schupp, E. (2000) Seed disperser effectiveness: the quantity component and patterns of seed rain for Prunus mahaleb. Ecological Monographs 70, 591-615.

Jordano, P., García, C., Godoy, J.A. and García-Castaño, J.L. (2007) Differential contribution of frugivores to complex seed dispersal patterns. Proceedings of the National Academy of Sciences USA 104, 3278-3282.

Julliot, C. (1997) Impact of seed dispersal of red howler monkeys Alouatta seniculus on the seedling population in the understorey of tropical rain forest. Journal of Ecology 85, $431-440$.

Kollmann, J. and Goetze, D. (1997) Notes on seed traps in terrestrial plant communities. Flora 192, 1-10.

Levey, D.J., Bolker, B.M., Tewksbury, J.J., Sargent, S. and Haddad, N.M. (2005) Effects of landscape corridors on seed dispersal by birds. Science 309, 146-148.

Loiselle, B. and Blake, J. (1993) Spatial distribution of understory fruit-eating birds and fruiting plants in a neotropical lowland wet forest. In: Fleming, T. and Estrada, A. (eds) Frugivory and Seed Dispersal: Ecological and Evolutionary Aspects. Kluwer Academic, Dordrecht, The Netherlands, pp. 177-189.

Mack, A. (1995) Distance and non-randomness of seed dispersal by the dwarf cassowary Casuarius bennetti. Ecography 18, 286-295.

Mack, A.L. and Druliner, G. (2003) A non-intrusive method for measuring movements and seed dispersal in cassowaries. Journal of Field Ornithology 74, 193-196.

Manzano, P. and Malo, J.E. (2006) Extreme long-distance seed dispersal via sheep. Frontiers in Ecology and the Environment 5, 244-248.

Marshall, T.C., Slate, J., Kruuk, L.E.B. and Pemberton, J.M. (1998) Statistical confidence for likelihood-based paternity inference in natural populations. Molecular Ecology 7, $639-655$.

McEuen, A.B. and Curran, L.M. (2004) Seed dispersal and recruitment limitation across spatial scales in temperate forest fragments. Ecology $85,507-518$.

Morales, J.M. and Carlo, T.A. (2006) The effects of plant distribution and frugivore density on the scale and shape of dispersal kernels. Ecology 87, 1489-1496.

Morin, P.A., Chambers, K.E., Boesch, C. and Vigilant, L. (2001) Quantitative polymerase chain reaction analysis of DNA from non-invasive samples for accurate microsatellite genotyping of wild chimpanzees (Pan troglodytes verus). Molecular Ecology 10, 1835-1844.

Muller-Landau, H., Wright, S., Calderón, O., Hubbell, S. and Foster, R. (2002) Assessing recruitment limitation: concepts, methods and case-studies from a tropical forest. In: Levey, D., Silva, W. and Galetti, M. (eds) Seed Dispersal and Frugivory: Ecology, Evolution and Conservation. CAB International, Wallingford, UK, pp. 35-53.

Murray, K. (1988) Avian seed dispersal of three neotropical gap-dependent plants. Ecological Monographs 58, 271-298.

Nakashizuka, T., lida, S., Masaki, T., Shibata, M. and Tanaka, H. (1995) Evaluating increased fitness through dispersal: a comparative study on tree populations in a temperate forest, Japan. Écoscience 2, 245-251.

Nathan, R. (2006) Long-distance dispersal of plants. Science 313, 786-788.

Nathan, R. and Muller-Landau, H.C. (2000) Spatial patterns of seed dispersal, their determinants and consequences for recruitment. Trends in Ecology and Evolution 15, 278-285.

Nathan, R., Horn, H., Chave, J. and Levin, S. (2002) Mechanistic models for tree seed dispersal by wind in dense forests and open landscapes. In: Levey, D., Silva, W. and 
Galetti, M. (eds) Seed Dispersal and Frugivory: Ecology, Evolution and Conservation. CAB International, Wallingford, UK, pp. 69-82.

Nathan, R., Perry, G., Cronin, J.T., Strand, A.E. and Cain, M.L. (2003) Methods for estimating long-distance dispersal. Oikos 103, 261-273.

Oddou-Muratorio, S., Petit, R., Le Guerroue, B., Guesnet, D. and Demesure, B. (2001) Pollen-versusseed-mediated gene flow in a scattered forest tree species. Evolution 55, 1123-1135.

Portnoy, S. and Willson, M. (1993) Seed dispersal curves: behaviour of the tail of the distribution. Evolutionary Ecology 7, 25-44.

Primack, R. and Levy, C. (1988) A method to label seeds and seedlings using gammaemitting radionuclides. Ecology 69, 796-800.

Queller, D. and Goodnight, K. (1989) Estimating relatedness using genetic markers. Evolution 43, 258-275.

Ramos-Fernandez, G., Mateos, J.L., Miramontes, O., Cocho, G., Larralde, H. and AyalaOrozco, B. (2004) Lévy walk patterns in the foraging movements of spider monkeys (Ateles geoffroyi). Behavioral Ecology and Sociobiology 55, 223-230.

Reid, N. (1989) Dispersal of mistletoes by honeyeaters and flowerpeckers: components of seed dispersal quality. Ecology 70, 137-145.

Rey, P. and Alcántara, J. (2000) Recruitment dynamics of a fleshy-fruited plant (Olea europaea): connecting patterns of seed dispersal to seedling establishment. Journal of Ecology 88, 622-633.

Sato, H. and Hiura, T. (1998) Estimation of overlapping seed shadows in a northern mixed forest. Forest Ecology and Management 104, 69-76.

Sato, T., Isagi, Y., Sakio, H., Osumi, K. and Goto, S. (2006) Effect of gene flow on spatial genetic structure in the riparian canopy tree Cercidiphyllum japonicum revealed by microsatellite analysis. Heredity 96, 79-84.

Schnabel, A., Nason, J. and Hamrick, J. (1998) Understanding the population genetic structure of Gleditsia triacanthos L.: seed dispersal and variation in female reproductive success. Molecular Ecology 7, 819-832.

Schupp, E.W., Howe, H.F., Augspurger, C.K. and Levey, D.J. (1989) Arrival and survival in tropical treefall gaps. Ecology 70, 562-564.

Schupp, E., Milleron, T. and Russo, S. (2002) Dissemination limitation and the origin and maintenance of species-rich tropical forests. In: Levey, D., Silva, W. and Galetti, M. (eds) Seed Dispersal and Frugivory: Ecology, Evolution and Conservation. CAB International, Wallingford, UK, pp. 19-33.

Skarpaas, O., Shea, K. and Bullock, J.M. (2005) Optimizing dispersal study design by Monte Carlo simulation. Journal of Applied Ecology 42, 731-739.

Snow, B. and Snow, D. (1988) Birds and berries. T. \& A.D. Poyser, Calton, UK.

Sun, C., Ives, A., Kraeuter, H. and Moermond, T. (1997) Effectiveness of three turacos as seed dispersers in a tropical montane forest. Oecologia 112, 94-103.

Taberlet, P. (1996) Reliable genotyping of samples with very low DNA quantities using PCR. Nucleic Acids Research 24, 3189 - 3194.

Valière, N. (2002) GIMLET: a computer program for analysing genetic individual identification data. Molecular Ecology Notes 2, 377-379.

Vander Wall, S.B. (2000) Secondary dispersal of jeffrey pine seeds by rodent scatterhoarders: the roles of pilfering, recaching and a variable environment. In: Levey, D., Silva, W. and Galetti, M. (eds) Seed Dispersal and Frugivory: Ecology, Evolution and Conservation. CAB International, Wallingford, UK, pp. 193-208.

Viswanathan, G.M., Bartumeus, F., Buldyrev, S.V., Catalan, J., Fulco, U.L., Havlin, S., da Luz, M.G.E., Lyra, M.L., Raposo, E.P. and Stanley, H.E. (2002) Lévy flight random searches in biological phenomena. Physica A 314, 208-213. 
Wang, B. and Smith, T. (2002) Closing the seed dispersal loop. Trends in Ecology and Evolution 17, 379-385.

Webb, C.O. and Peart, D.R. (2001) High seed dispersal rates in faunally intact tropical rain forest: theoretical and conservation implications. Ecology Letters 4, 491-499.

Wenny, D. and Levey, D. (1998) Directed seed dispersal by bellbirds in a tropical cloud forest. Proceedings of the National Academy of Sciences USA 95, 6204-6207.

Westcott, D.A. and Graham, D.L. (2000) Patterns of movement and seed dispersal of a tropical frugivore. Oecologia 122, 249-257.

Westcott, D.A., Bentrupperbaumer, J., Bradford, M.G. and McKeown, A. (2005) Incorporating patterns of disperser behaviour into models of seed dispersal and its effects on estimated dispersal curves. Oecologia 146, $57-67$.

Whitney, K., Fogiel, M., Lamperti, A., Holbrook, K., Stauffer, D., Hardesty, B., Parker, V. and Smith, T. (1998) Seed dispersal by ceratogymna hornbills in the Dja Reserve, Cameroon. Journal of Tropical Ecology 14, 351-371.

Wilson, G.A. and Rannala, B. (2003) Bayesian inference of recent migration rates using multilocus genotypes. Genetics 163, 1177-1191.

Yumoto, T. (1999) Seed dispersal by Salvin's curassow, Mitu salvini (Cracidae), in a tropical forest of Colombia: direct measurements of dispersal distance. Biotropica 31, 654-660.

Ziegenhagen, B., Liepelt, S., Kuhlenkamp, V. and Fladung, M. (2003) Molecular identification of individual oak and fir trees from maternal tissues of their fruits or seeds. Trees Structure and Function 17, 345-350. 